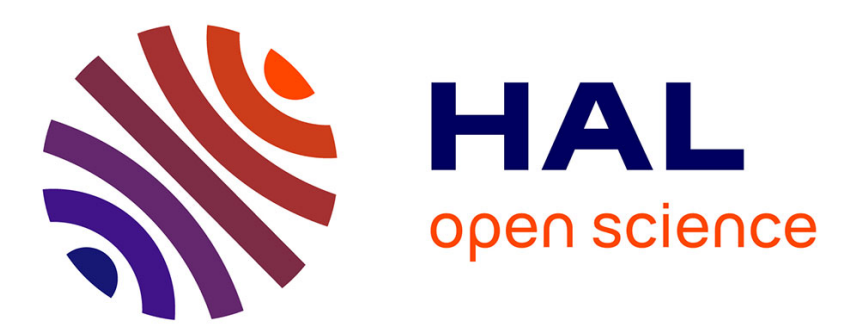

\title{
Observations sur un aspect de la variabilité constatée au cours de la multiplication végétative de variétés de vigne issues de semis de Vitis vinifera $L$
} Serge Grenan, Paul Truel

\section{- To cite this version: \\ Serge Grenan, Paul Truel. Observations sur un aspect de la variabilité constatée au cours de la multiplication végétative de variétés de vigne issues de semis de Vitis vinifera L. Agronomie, 1983, 3 (7), pp.675-680. hal-00884558}

\section{HAL Id: hal-00884558 https://hal.science/hal-00884558}

Submitted on 1 Jan 1983

HAL is a multi-disciplinary open access archive for the deposit and dissemination of scientific research documents, whether they are published or not. The documents may come from teaching and research institutions in France or abroad, or from public or private research centers.
L'archive ouverte pluridisciplinaire HAL, est destinée au dépôt et à la diffusion de documents scientifiques de niveau recherche, publiés ou non, émanant des établissements d'enseignement et de recherche français ou étrangers, des laboratoires publics ou privés. 


\section{Observations sur un aspect de la variabilité constatée au cours de la multiplication végétative de variétés de vigne issues de semis de Vitis vinifera L.}

Serge GRENAN - Paul TRUEL*

Association Nationale Technique pour l'Amélioration de la Viticulture (ANTAV), Domaine de l'Espiguette, F 30240 Le Grau-du-Roi.

* I.N.R.A., Station de Recherches de Viticulture, Domaine de Vassal, Centre de Recherches de Montpellier, 34340 Marseillan.

Chez la vigne, les variétés obtenues par multiplication végétative d'un seul individu issu de la germination d'une graine sont génétiquement homogènes. Cependant certains individus de ces variétés peuvent présenter au cours des multiplications des modifications morphologiques. Ces variations du comportement ont été particulièrement étudiées sur la variété de raisins de table « Danam B. ». Cette variabilité est rapprochée du mode de fonctionnement juvénile connu chez la vigne.

Mots clés additionnels : Plant de semis, type "rouge", type "vert ", fonctionnement juvénile, Polymorphisme.

Varieties obtained by vegetative propagation from one seedling are genetically homogeneous. However, during multiplication morphological modifications may be observed in some plants of these new varieties. These variations were studied on the table grapevine variety « Danam $B$ ». This variability is compared with known juvenile behaviour in grapevine.

Additional key words : Seedling, "red" type, "green" type, juvenility, polymorphism.

\section{INTRODUCTION}

La culture de la vigne à fruits, Vitis vinifera L., s'est étendue en France essentiellement à partir de populations issues de semis spontanés mais aussi à partir de variétés en provenance des divers pays du bassin méditerranéen introduites au cours des siècles. La première origine trouve une confirmation dans la possibilité de regrouper les différentes variétés en familles ampélographiques rattachées à leur zone de culture, dans la mesure où celle-ci coïncide avec leur zone d'origine (LEVADOUX, 1956).

En viticulture, la variété, appelée aussi cépage, qui a été distinguée empiriquement par les praticiens, a une valeur agronomique très importante. Elle peut être définie comme un ensemble d'individus maintenus par multiplication végétative, présentant des aptitudes communes sur le plan cultural et technologique mais susceptibles de montrer une certaine variation sur le plan morphologique et parfois phénologique, sans toutefois entraîner des différences importantes; c'est donc une variété population.

Au cours de l'extension de la culture de la vigne, cette variabilité a été remarquée par les viticulteurs qui ont essayé de distinguer des types variétaux à l'intérieur des différents cépages. Depuis une trentaine d'années, ce polymorphisme est à la base de la sélection clonale. Celle-ci est possible pour les variétés populations mais il est généralement admis qu'elle est inefficace à l'intérieur de variétés clones obtenues par multiplication végétative d'un seul individu provenant de la germination d'une graine, sauf dans le cas où l'aspect sanitaire est seul pris en compte. Les variétés «Alphonse Lavallée N. » et « Cardinal R. », par exemple, sont considérées comme génétiquement homogènes. Or des observations récentes semblent montrer que, pour ces variétés clones, il peut apparaître des différences morphologiques entre individus, telles que celles que nous décrivons dans cet article. 


\section{DESCRIPTION DES VARIATIONS MORPHOLOGIQUES}

La compréhension des phénomènes étudiés nécessite au préalable de décrire succinctement un mode de comportement au vignoble de jeunes individus issus de croisements entre variétés de $V$. vinifera durant les $1^{\text {res }}$ annécs de végétation.

Génćralement les populations de plants, issus de ce type de croisement, sont caractérisées par une forte découpure des feuilles. Cettc lobation s'accompagne fréquemment d'une grande densité de poils dressés sur la face inféricure du limbe et d'une intense pigmentation anthocyanique des ramcaux, des jeunes feuilles, des pétioles et des nervures des feuilles adultes, ainsi que des pédoncules des grappes.

Les individus à feuilles très découpées et pubescentes et à organes végétatifs fortement pigmentés sont plus fréquents dans ces populations de semis que dans l'ensemble des varićtés cultivées de $V$. vinifera ou des jeunes plants qui en sont issus par multiplication végétative.

Cette différence peut être la conséquence d'une sćlection qui a éliminé les formes les plus découpées, mais on peut également penser qu'elle est le résultat d'une évolution de la morphologie des plantes. En effet, la manifestation d'un tel

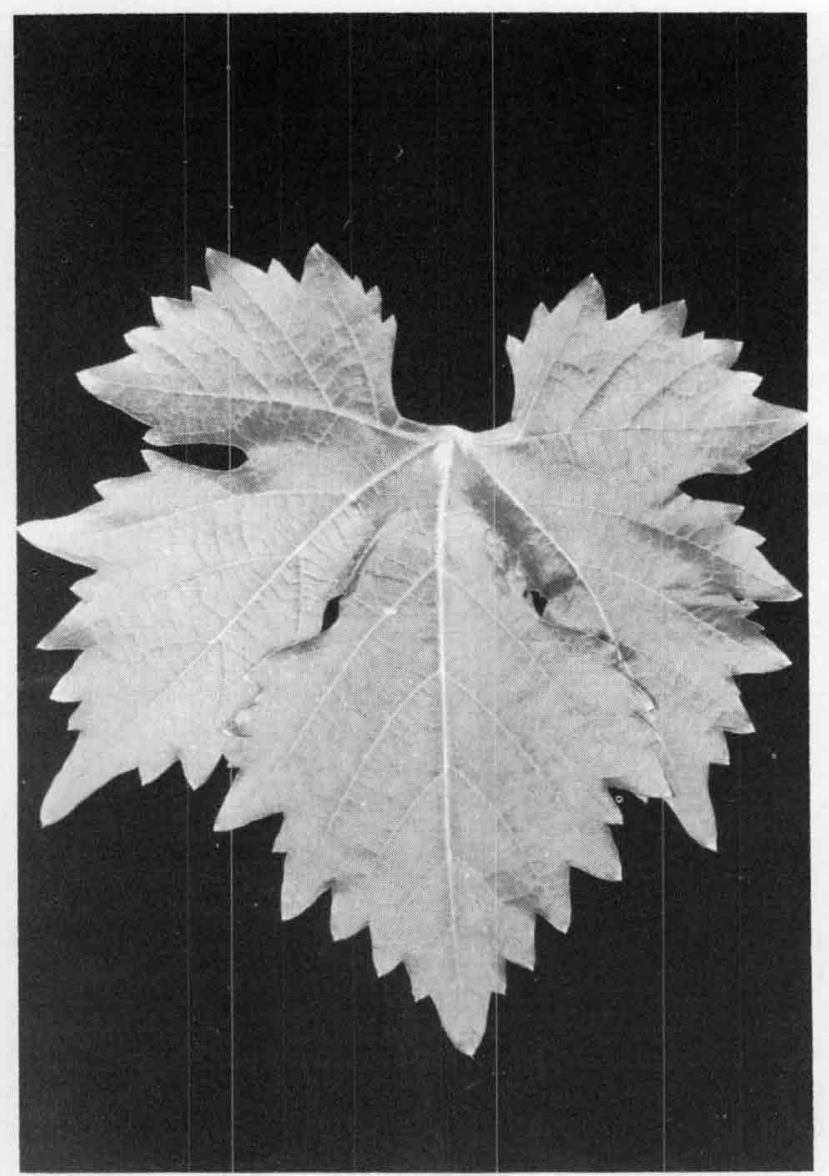

Figure 1

Comparaison de feuilles de "Danam B."

a) Féuille découpée de "Danam B." type "rouge ».

b) Fenille entière de "Danam B. " type "vert".

tuent le matéric de départ à partir duquel ont été réalisées diverses descendances par multiplication végétative.

- la multiplication par bouturage (en 1973-1974) de 2 de ces souches a permis de constituer 2 familles comptant respectivement 20 et 8 ceps développés et effectivement observés (tabl. 1). phénomène de modification de la morphologie foliaire au cours du temps a été observée sur divers cépages issus de croisements entre varićtés de $V$. vinifera.

\section{A. Variété « Danam B. »}

La variété «Danam B. » (Dabouki B. $\times$ Muscat de Hambourg N. - croisement de 1958), qui est constituée uniquement par les souches issues de la multiplication végétative d'un scul cep provenant de la germination d'une graine, présente ainsi la particularité de posséder des souches dont la morphologie est non seulement différente, mais peut aussi sc modificr d'unc année à l'autre.

La souche mère ayant été détruite, il n'est pas possible de connaitre sa morphologic exacte.

Les 5 souches qui en sont issues par multiplication végétative (bouturage dans l'hiver 1966-1967) ont toutes actuellement des rameaux rouges portant des feuilles quinquclobées, profondément découpćes, pubescentes, à nervures et pétiole rouges, avec lc pédoncule et l'axe de la rafle fréquemment teintés de rouge. Ces 5 souches, d'aspect homogène, à découpure prononcéc et à pigmentation intense sont dites du type «rouge » (fig. 1a). Elles consti-

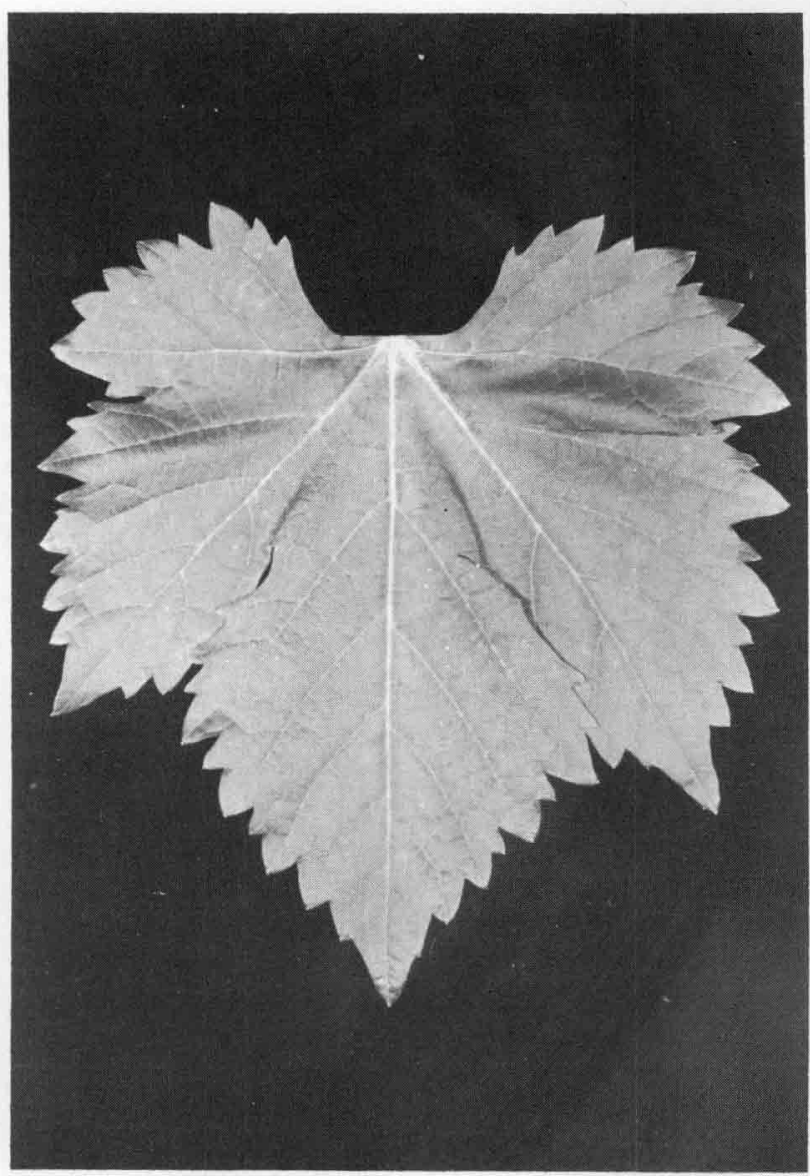

Comparaison of "Danam B. "leaves.

a) Leaf with deep sinuses of "red" type "Danam B."

b) Leaf with shallow sinuses of "green" type "Danam B. "

- Dans la $1^{\text {re }}$ famille, issue de la souche $n^{\circ} 1$, on observait en 1978 (4`annéc de végétation) la présence de 4 individus à rameaux plus verts (faiblement pigmentés), avec des feuilles moins découpées, moins pubescentes et à nervures vertes. Ces souches sont dites du type « vert» (fig. $1 b$ ). En 1979 et 1980, cettc observation ćtait confirmée et en 1981 
une $5^{\mathrm{e}}$ souche «vert » apparaissait en plus des précédentes (tabl. 2).

- Dans la $2^{c}$ famille issue de la souche $n^{\circ} 2$, on notait la présence d'une souche à rameaux verts en 1980 et de 2 en 1981 (tabl. 2).

- unc multiplication par greffage, en 1976, de 3 des 5 souches de départ a donné 3 familles: $s 1, s 2, s 4$. Le portegreffe utilisé est un clone de la variété «SO4» (V. Berlandieri $\times \mathrm{V}$. riparia). Après 4 années de végétation, la répartition des plants est la suivante:

1) Dans la famille issue de la souche $n^{\circ} 1,60$ ceps étaient assez développés en 1980 pour effectuer ainsi leur classement : 23 individus de type "vert " et 37 de type "rouge ».

\section{TABLEAU 1}

Multiplications successives de la variété "Danam B. » Successive propagations of "Danam B."

\begin{tabular}{|c|c|c|}
\hline & Année & $\begin{array}{c}\text { Nombre } \\
\text { de souches plantées }\end{array}$ \\
\hline Croisement & 1958 & \\
\hline Plantation souche mère & $1961-1962$ & 1 \\
\hline $\begin{array}{l}\text { Bouturage de la souche } \\
\text { mère }\end{array}$ & e $1966-1967$ & \\
\hline $\begin{array}{l}\text { Plantation du matériel de } \\
\text { départ }\end{array}$ & $1967-1968$ & $5(\mathrm{~s} 1, \mathrm{~s} 2, \mathrm{~s} 3, \mathrm{~s} 4, \mathrm{~s} 5)$ \\
\hline $\begin{array}{l}\text { Bouturage du matériel de } \\
\text { départ }\end{array}$ & 1973-1974 & \\
\hline Plantation & $1974-1975$ & $\mathrm{~s} 1 \rightarrow 20 \quad \mathrm{~s} 2 \rightarrow 10$ \\
\hline $\begin{array}{l}\text { Greffage sur table du } \\
\text { matériel } \\
\text { de départ }\end{array}$ & 1976 & \\
\hline Plantation & 1976-1977 & $\mathrm{s} 1 \rightarrow 78 \quad \mathrm{~s} 2 \rightarrow 30 \quad \mathrm{~s} 4 \rightarrow 11$ \\
\hline
\end{tabular}

TABLEAU 2

Evolution morphologique au cours des années ( $R$ : rouge; $V:$ vert). Morphological evolution during the years ( $R:$ red; $V:$ green).

\begin{tabular}{ccrrrrr}
\hline \hline & \multicolumn{3}{c}{ Bouturage } & \multicolumn{3}{c}{ Greffage } \\
\hline \multirow{2}{*}{ Année } & Type & S 1 & S 2 & S 1 & S 2 & S 4 \\
\hline \multirow{2}{*}{1978} & $\mathrm{R}$ & 16 & 8 & & & \\
& $\mathrm{~V}$ & 4 & - & & & \\
\hline \multirow{2}{*}{1979} & $\mathrm{R}$ & 16 & 8 & & & \\
& $\mathrm{~V}$ & 4 & - & & & \\
\hline \multirow{2}{*}{1980} & $\mathrm{R}$ & 16 & 7 & 37 & 15 & 11 \\
& $\mathrm{~V}$ & 4 & 1 & 23 & 8 & - \\
\hline \multirow{2}{*}{1981} & $\mathrm{R}$ & 15 & 6 & 29 & 7 & 10 \\
& $\mathrm{~V}$ & 5 & 2 & 31 & 16 & 1 \\
\hline \hline
\end{tabular}

En 1981, 8 souches classées dans le type « rouge » l'année précédente, sont apparues de type « vert " (tabl. 2).

2) Dans la famille issue de la souche $n^{\circ} 2$, dont 23 ceps étaient assez développés pour être classés dans l'un des deux types, on notait en 1980 la présence de 8 individus de type "vert " et 15 de type « rouge ». En 1981, 8 souches notées de type «rouge » l'année précédente étaient rattachées au type « vert » (tabl. 2).
Il doit être précisé que 3 de ces souches semblaient présenter en 1981 les 2 types avec toutefois une prédominance du typc «vert». En particulier une d'entre-elles portait des rameaux du type « rouge" sur le courson (base d'un sarment qui après la taille porte 2 yeux) et du type «vert» sur le long bois (sarment taillé plus long qui porte généralement de 6 à 10 yeux).

3) Enfin la famille issue de la souche $n^{\circ} 4$, représentée par 11 ceps, ne comptait en 1980 aucune souche du type «vert». En 1981, une souche était classée dans ce type, tandis qu'une autre, maintenue dans le type "rouge", paraissait cependant moins pigmentée que les autres (tabl. 2).

Ainsi le changement se fait en général du type « rouge » vers le type «vert» lequel est le plus souvent stable. Cependant des oscillations autour de l'état rouge-vert peuvent apparaître au cours des observations, car les différents niveaux d'insertion des rameaux sur le cep rendent parfois difficile le classement des souches.

C'est ainsi que les notations pratiquées en 1982 ont conduit à classer dans le type "rouge " des souches qui avaient été rattachées au type «vert» au cours des 2 années précédentes. Ce changement apparent dans le sens de la variation peut être rapproché des observations effectuées en 1981 et concernant la présence sur une même plante des 2 types en fonction du système de taille. En effet, des opérations telles que la taille peuvent plus ou moins favoriser l'expression de l'un de ces types et expliquer les différents classements. De même, les conditions climatiques et leur action sur le développement de chaque individu peuvent avoir une influence, variable dans certaines limites, sur la pigmentation des rameaux. Ces considérations montrent la nécessité d'examiner plus précisément le cas des individus qui ont eu un comportement différent en 1982 et d'étudier en particulier les relations pouvant exister entre la taille, la vigueur et l'expression de types morphologiques.

En résumé, les observations faites sur les descendances végétatives de ce plant do scmis montrent que la multiplication de souches de type "rouge " a donné un certain nombre de souchés de type "vert». Ainsi, dans le cas du greffage sur 94 plants observés pendant 3 annécs consécutives :

50 individus notés rouges en 1980 n'ont pas changé

24 individus notés verts en 1980 n'ont pas changé 1982

5 individus notés rouges en 1980 sont verts en 1981 et

15 individus notés verts en 1980 ou 1981 sont redevenus rouges en 1982

Les 24 individus qui ont été notés verts en 1980 peuvent être apparus, soit à la suite d'une évolution comparable à celle qui a été observée sur les 5 individus rouges en 1980 et verts en 1981 et 1982, soit dès l'année du greffage ce qui laisserait supposer qu'une modification s'était déjà produite dans le greffon prélevé sur la souche-mère.

\section{B. Variété Lival N.}

Un autre exemple de modifications morphologiques de cette nature a été observé chez la variété «Lival N. » (Alphonse Lavallée N. $\times$ Lignan B. - croisement de 1956).

Dans ce cas, le type «vert » ne correspond pas à une absence de coloration anthocyanique des organes végétatifs. Ainsi les individus de ce type ont une pigmentation atténuée par rapport au type « rouge», donnant aux rameaux une couleur rouge moins intense. Les feuilles des individus du type « vert » ont une pubescence qui s'atténue sans disparaître et la différence dans la découpure des feuilles n'est pas 
assez marquée pour être utilisée cornme caractère distinctif.

Des 5 souches issues de la multiplication par bouturage du plant de semis (détruit) du "Lival N.", 3 sont de type «rouge" et 2 de type «vert». Contrairement donc au «Danam B. », la descendance provenant de la $1^{\text {rc }}$ multiplication du «Lival N. " n'est actuellement pas de type homogène. Une multiplication par greffage sur un clone du porte-greffe $\mathrm{SO} 4$ des 5 souches de départ a donné, d'une part, une descendance homogène de type « rouge » pour les 3 souches de ce type et, d'autre part, une descendance de type « vert » pour les 2 souches de ce type.

Ainsi pour cette variété, il a seulement été constaté l'apparition d'individus de type « vert " après la $1^{\text {rc }}$ multiplication, mais il n'a pas été observé de variation d'un typc vers l'autre au cours des multiplications ultérieures.

\section{DISCUSSION}

Il nous a paru intéressant de tenter un rapprochement cntre les variations que nous avons observécs et divers stades du développement de la vigne à partir de la germination d'une graine.

Le jeune plant fonctionne d'abord selon le mode juvćnile, caractérisé par une phyllotaxic spiralće et par l'incapacité à former des vrilles. Cette courte période (6 à 12 nœuds) correspond à la phase juvénile proprement dite. Puis la tige acquiert rapidement un mode de fonctionnement différent caractérisé par une phyllotaxie distique et la production de vrilles. Dans les conditions normales de culture au vignoble, la vigne continue de se développer selon ce mode et généralement les $1^{\text {res }}$ inflorescences apparaissent au bout de 2 ou 3 ans. On peut considérer la période corrcspondant à l'absence de formation d'organes reproducteurs comme une phase de jeunesse de la vigne dont la durée diminue lorsque la vigueur augmente et qui peut être également raccourcie par les techniques particulières de culture en serre et de taille des plants de semis (HUGLIN \& JULLIARD, 1964 ; WAGNER \& BRONNER, 1974).

Cette phase exclusivement végétative peut être rapprochée du type morphologique à pigmentation anthocyanique plus marquée et à feuilles plus découpées et plus pubescentes, qui se manifeste dans une $1^{\text {rc }}$ étape du développement de certains plants de semis.

Cependant, nous avons vu que ces caractéristiques morphologiques se perpétuent sur certains individus après la formation des $1^{\text {rcs }}$ grappes. L'apparition d'une morphologie de type adulte et l'initiation d'organes reproducteurs sont 2 phénomènes indépendants. Le fonctionnement morphologique de type adulte, d'après les descriptions précédentes, pourrait être caractérisé par une lobation foliaire plus faible, une pubescence discrète ou nulle et une pigmentation atténuée. Ainsi, chez la vigne, certains traits de jeunesse persisteraient plus ou moins longtemps après le début de la phase reproductive. Le passage, sur le plan morphologique, d'un fonctionnement de type juvénile « rouge " vers un type adulte « vert " pourrait s"effectuer à des moments divers selon les individus et selon les cépages.

En fait, il est possible que sur de nouvelles variétés issues de semis, le stade juvénile disparaisse très vite et la stabilité morphologique s'installe rapidement. C'est ainsi que sur la variété «Ribol N." (Olivette B. $\times$ Alphonse Lavallée N. - croisement de 1958) à rameau vert portant des feuilles peu découpées, il n'a jamais été vu de souches ayant les caractéristiques de rameau rouge portant des feuilles découpées. L'observation depuis 5 années de 120 individus issus d'une $1^{\text {rc }}$ multiplication par bouturage suivi d'un greffage a permis de constater l'homogénéité et la stabilité du type «vert».

Par contre, une prolongation de la phase de jeunesse a pu être observéc sur la variété "Cardinal $R$. " cultivée non greffée, (VAlat, comm. pers.). De même, la présence de types «rouge» et «vert» chez la variété «Alphonse Lavalléc N. " peut ĉtre rattachée à ces phénomènes (TRUEL \& VALAT, 1971). Les observations au vignoble sur ce cépage montrent que les individus de type « rouge» n'existent plus qu'à l'état de traces dans les plantations. On peut penser que leur disparition est la conséquence d'unc évolution ou d'une sélection par les viticulteurs, ce qui impliquerait dans ce dernier cas une relation entre les caractéristiques morphologiques et d'autres caractères comme la production ou la vigucur. Pour vérifier cette éventualité, des mesures sont en cours sur la variété «Danam B.». Si une telle relation était démontrće, elle aurait des répercussions sur le plan agronomique et sur la pratique de la sélection.

Il peut être intéressant de signaler qu'une relation analogue, cntre le type morphologique et la capacité à former des grappes, peut se rencontrer dans des situations différentes de celles que nous avons étudiées, sans que l'on puisse pour autant rattacher cette relation à un type de fonctionnement particulier.

C'est ainsi que pour certains cépages populations très polymorphes, divers auteurs distinguent un type "rouge", à rameaux fortement pigmentés, à feuilles très découpées et peu productif, et un type «vert », à rameaux peu pigmentés, à feuilles entières et plus productif. Dans certains cas extrêmes, les caractéristiques ampélographiques peuvent servir de critère discriminatoire pour le choix des clones au cours de la sélection.

Ces variations morphologiques plus ou moins liées avec les potentialités fructifères ont été observées sur plusieurs cépages. On peut citer quelques-uns d'entre eux qui ont été particulièrement étudiés: "Chasselas B. » (LEVADOUX, 1951 ; BISSON, 1956 ; RIVES et al., 1974 ; SIMON comm. pers.), «Chenin B. » (Guillot comm. pers. ; Rouquie \& Remoue, 1981), «Pinot N.» (Bernard, 1981), «Sauvignon B. " (LAVIGNAC comm. pers.), "Savagnin B. " (BAlthazARD et al., 1976), "Sémillon B. " (LeClaiR comm. pers.).

De même, ce comportement des plants issus de semis peut être rapproché de la morphologie particulière des gourmands qui se présentent sous un type «rouge» à feuilles très découpées et qui sont généralement stériles, du moins l'annce de leur formation. Le fonctionnement juvénile des gourmands peut s'expliquer par leur origine située dans une zone proche du système racinaire et par le fait qu'ils naissent à partir des bourgeons longtemps inhibés. Ces 2 conditions, d'après NOZERAN \& RossignOLBANCILHON (1977) et NOZERAN et al. (1982), conduisent à des processus de rajeunissement ou de conservation du mode de fonctionnement juvénile.

Il est à noter que, sur la variété « Danam B.", les individus de type «vert " émettent des gourmands caractérisés par une forte pigmentation et une découpure prononcée des feuilles.

Enfin, dans les conditions expérimentales, on peut induire des modifications morphogénétiques de même nature. Les techniques de culture in vitro entraînent une miniaturisation des pousses qui fonctionnent selon le mode juvénile (phyllotaxic rayonnante et absence de vrilles). Les plants issus de multiplication in vitro, placés dans les conditions du vignoble, se comportent de façon différente 
de celle du pied-mère. Des modifications morphologiques apparaissent à des degrés divers sur l'ensemble des ceps ainsi cultivés. Les organes les plus constamment et durablement modifiés sont les feuilles qui sont très découpées et pubescentes et les rameaux qui ont une pigmentation anthocyanique. Certaines variétés peuvent devenir presque stériles. Ces particularités, caractéristiques de la phase de jeunesse peuvent être artificiellement maintenues par les pratiques culturales traditionnelles comme la taille courte qui entretient les caractéristiques d'un fonctionnement juvénile induites par le passage in vitro. Au contraire, la taille longue peut conduire à un « retour » vers un fonctionnement de type adulte (GRENAN, 1982).

L'ensemble de ces constatations sur les nouvelles variétés soulève quelques problèmes relatifs à leur sélection, leur multiplication et leur identification. Les questions qui peuvent être posées sont les suivantes : comment obtenir le plus rapidement possible une forme stable et vérifier cette stabilité ? quelle peut être l'incidence pratique de la coexistence de 2 types morphologiques dans une nouvelle variété ?

Sur le $1^{\text {cr }}$ point, on peut penser que la technique mise en œuvre par HUGLIN \& JULLIARD (1964) et apppliquéc par WAGNER \& BRONNER (1974) qui permet d'atteindre rapidement la phase de fructification, doit également favoriser le déploiement rapide de la morphogenèse. S'il en est ainsi la multiplication devrait être assurée en utilisant le matéricl prélevć soit à l'extrémité de la tige dans les cas de greffage et de bouturage du plant de semis, soit à l'extrémité des sarments de la souche mère dans le cas de sa transplantation directe.
Sur le $2^{\mathrm{c}}$ point, la multiplication des scules souches du type "vert " devrait ctre entreprise dans la mesure où il sera prouvé qu'elle assure la production de matériel homogène et stable.

\section{CONCLUSION}

Nos premières observations ont mis l'accent sur la présence au vignoble d'individus fonctionnant de nombreuses ánnées sclon le mode juvénile et se développant à côtć d'individus ayant acquis une morphogenèse caractéristique de l'état adulte. La concomitance de ces 2 types de fonctionnement tend à montrer que, chez $V$. vinifera, la phase que nous avons appelée de jeunesse n'est pas nécessairement fugace contrairement à une opinion généralement admise.

L'ensemble de ces remarques ne permettent plus, nous semble-t-il, d'interpréter les phénomènes que nous avons observés sur les cépages clones comme le résultat de mutations, mais elles incitent à penser que ces modifications sont la marque d'unc évolution dans la morphogenèse normale de la plante qui conduit à l'installation du mode de fonctionnement adulte.

Reçu le 29 juin 1982. Accepté le 9 mars 1983

\section{REMERCIEMENTS}

Nous remercions M. Le Professeur Nozeran - Faculté des Sciences d'Orsay - de nous avoir permis de replacer nos observations dans le cadre de la morphogenèse de la vigne.

\section{RÉFÉRENCES BIBLIOGRAPHIQUES}

Balthazard J., Arbault B., Levaux J., 1976. Exemple de sélection dans un cépage hétérogène: le Savagin Blanc du Jura. 2nd Symposium international sur la sélection clonale de la Vigne, Domaine de l'Espiguette (10 et 11 sept.).

Bernard R., 1981. La sćlection clonale. Le Vigneron champenois, 12, 505-511.

Bisson J., 1956. Etude ampélographique de populations de Chasselas du Tarn et Garonne. Proğ. agric. et vitic., 6-7, 85-95.

Grenan S., 1982. Quelques réflexions à propos des modifications morphogénétiques consécutives à la culture in vitro de la vigne (Vitis vinifera L.). Ann. Sci. nat. Bot., 4, 135-146.

Huglin P., Julliard B., 1964. Sur l'obtention de semis de vigne très vigoureux à misc à fruit rapide ct ses répercussions sur l'amélioration génétique de la vigne. Ann. Amélior. Plantes, 14 (3), 229-244. Levadoux L., 1951. La sélection et l'hybridation chez la Vigne. Ann. Ec. natl. sup. Agric. Montpellier, 28, 195 p.

Levadoux L., 1956. Les populations sauvages et cultivées de Vitisvinifera L. Ann. Amélior. Plantes, 1, 59-118.
Nozeran R., Rossignol-Bancilhon L., 1977. La multiplication végétative chez les végétaux vasculaires. Coll. Morphogenèse, Orsay Bull. Soc. Bot. Fr., 124, 59-96.

Nozeran R., Ducreux G., Rossignol-Bancilhon L., 1982. Réflexions sur les problèmes de rajcunissement chez les végétaux. Bull. Soc. Bot. Fr., 129, Lettres botaniques 1982, 2, 107-130.

Rives M., Leclair P., Hevin M., 1974. La sélection clonalc du Chasselas de Moissac. L'action agricole du Tarn-et-Garonne, 454 bis, 5-13.

Rouquie P., Remoue M., 1981. La sćlcction clonale de la vigne dans le Val-de-Loire. C.R. Acad. Agric. Fr., 115-132.

Truel, Valat C., 1971. A propos de l'Alphonse Lavalléc. Prog. agric. et vitic., 24, 513-515.

Wagner R., Bronner A., 1974. Etude de la fertilité de semis de Vitis vinifera: application à la mise au point d'un test précoce de sélcction. Ann. Amélior. Plantes, 24 (2), 145-157. 